Supporting Information

\title{
Understanding of Face-on Crystallites Transitioning to Edge-on Crystallites in Thiophene-based Conjugated Polymers
}

Sung Yun Son, ${ }^{\mathrm{a}, \mathrm{b}}$ Taiho Park ${ }^{*, a}$ and Wei You*,b

${ }^{a}$ Department of Chemical Engineering, Pohang University of Science and

Technology (POSTECH), Pohang, Gyeongbuk, 37673, Korea

${ }^{b}$ Department of Chemistry, University of North Carolina at Chapel Hill, Chapel Hill, North Carolina 27599, United States

E-mail: taihopark@postech.ac.kr and wyou@unc.edu 


\section{Detailed synthetic procedures}

Synthesis of P3HT. In a glove box under $\mathrm{N}_{2}$ atmosphere, $2.0 \mathrm{M}$ of isopropylmagnesium chloride in THF $(0.5 \mathrm{ml}, 1 \mathrm{mmol})$ was added to a solution of 2,5-dibromo-3-hexylthiophene (326 $\mathrm{mg}, 1$ $\mathrm{mmol})$ in anhydrous THF $(20 \mathrm{ml})$, and the reaction mixture was stirred for $1 \mathrm{~h}$ at room temperature. Then, $\mathrm{Ni}(\mathrm{dppp}) \mathrm{Cl}_{2}(5.6 \mathrm{mg}, 0.0103 \mathrm{mmol})$ dispersed in THF $(5 \mathrm{~mL})$ was added and the reaction mixture was stirred for $3 \mathrm{~h}$. After that time period, the reaction mixture was taken out of the glove box and quenched by adding $5 \mathrm{~N} \mathrm{HCl}$. The reaction mixture was poured into methanol, then the crude polymer was collected via filtration, which was then purified by Soxhlet extraction with methanol, hexane, and chloroform. The polymer solution in chloroform was concentrated under reduced pressure/rotovap, and the polymer was precipitated into methanol. The polymer was collected via filtration, which was then dried in vacuum oven for $24 \mathrm{~h}$ at an ambient temperature (126 mg, $76 \%$ ). GPC: $\mathrm{M}_{\mathrm{n}}=16,800, Ð=1.07 .{ }^{1} \mathrm{H}-\mathrm{NMR}\left(500 \mathrm{MHz}, \mathrm{CDCl}_{3}, \mathrm{ppm}\right): \delta 6.98(\mathrm{~s}, 1 \mathrm{H})$; $2.81(\mathrm{t}, J=7.8 \mathrm{~Hz}, 2 \mathrm{H}) ; 1.71(\mathrm{~m}, 2 \mathrm{H}) ; 1.44(\mathrm{~m}, 2 \mathrm{H}), 1.35(\mathrm{~m}, 4 \mathrm{H}) ; 0.91(\mathrm{t}, J=6.6 \mathrm{~Hz}, 3 \mathrm{H})$.

Synthesis of RP-3T. In a glove box under $\mathrm{N}_{2}$ atmosphere, $2.0 \mathrm{M}$ of isopropylmagnesium chloride in THF $(0.81 \mathrm{ml}, 1.62 \mathrm{mmol})$ was added to a solution of 2,5-dibromo-3-hexylthiophene (489 $\mathrm{mg}$,

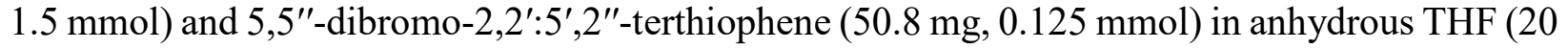
$\mathrm{ml})$, and the reaction mixture was stirred for $1 \mathrm{~h}$ at room temperature. Then, $\mathrm{Ni}(\mathrm{dppp}) \mathrm{Cl}_{2}(9.5 \mathrm{mg}$, $0.0175 \mathrm{mmol})$ dispersed in THF $(5 \mathrm{~mL})$ was added and the reaction mixture was stirred for $3 \mathrm{~h}$. After that time period, the reaction mixture was taken out of the glove box and quenched by adding $5 \mathrm{~N} \mathrm{HCl}$. The reaction mixture was poured into methanol, then the crude polymer was collected via filtration, which was then purified by Soxhlet extraction with methanol, hexane, and chloroform. The polymer solution in chloroform was concentrated under reduced pressure/rotovap, and the polymer was precipitated into methanol. The polymer was collected via filtration, which was then dried in vacuum oven for $24 \mathrm{~h}$ at an ambient temperature $(218 \mathrm{mg}, 65 \%)$. GPC: $\mathrm{M}_{\mathrm{n}}=15,200, Ð$ $=1.38 .{ }^{1} \mathrm{H}-\mathrm{NMR}\left(500 \mathrm{MHz}, \mathrm{CDCl}_{3}, \mathrm{ppm}\right): \delta 7.18-7.04(\mathrm{br}, 0.59 \mathrm{H}) ; 7.04-6.92(\mathrm{br}, 1 \mathrm{H}) ; 2.81(\mathrm{t}, J$ $=7.8 \mathrm{~Hz}, 2 \mathrm{H}) ; 1.71(\mathrm{~m}, 2 \mathrm{H}) ; 1.44(\mathrm{~m}, 2 \mathrm{H}), 1.35(\mathrm{~m}, 4 \mathrm{H}) ; 0.91(\mathrm{t}, J=6.6 \mathrm{~Hz}, 3 \mathrm{H})$. 
Figure S1. ${ }^{1} \mathrm{H}$ NMR spectrum of RP-3T.

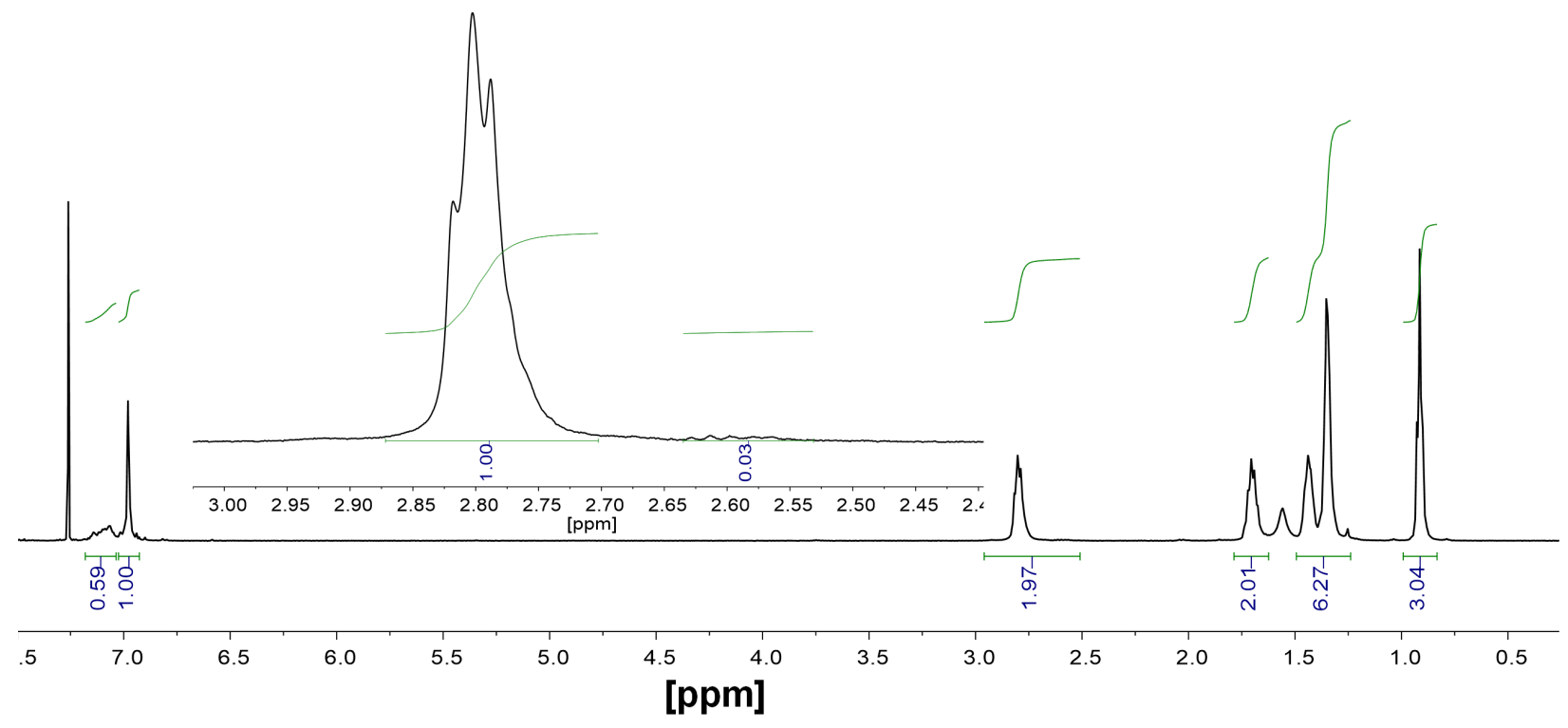

Figure S2. ${ }^{1} \mathrm{H}$ NMR spectrum of P3HT.

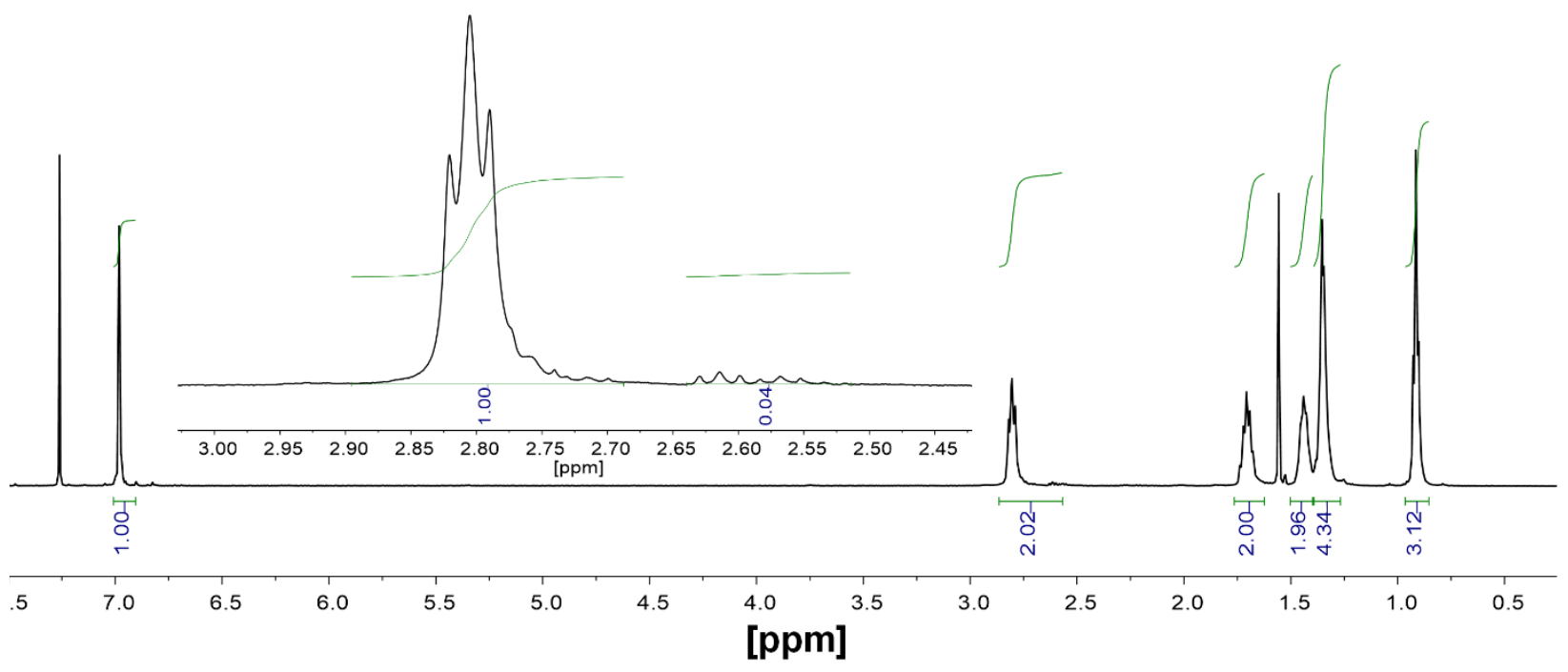


Figure S3. (a) Pole figures for the (100) and (010) peaks from RP-3T films annealed at 150, 160, 170,180 , or $190^{\circ} \mathrm{C}$ for $10 \mathrm{~min}$. The pole figures are drawn from the right half of the GIWAXS patterns. It should be noted that the peaks start from $2^{\circ}$ and $10^{\circ}$ in the pole figures for the (100) and (010) peaks, respectively, due to the curvature of the Ewald sphere giving rise to the inaccessible wedge in the out-of-plane direction. (b) Relative population of the crystallites with a particular orientation $\chi$, the polar angle, for (100) and (010) peaks from RP-3T annealed films. The relative population of the crystallites was obtained by performing geometrical correction, $\sin (\chi) \mathrm{I}(\chi)$, on the pole figures. In the plot for the (100) peak, $\chi=0-30^{\circ}\left(\chi=60-90^{\circ}\right)$ indicates edge-on (face-on) orientation, while in the plot for the (010) peak, $\chi=0-30^{\circ}\left(\chi=60-90^{\circ}\right)$ indicates faceon (edge-on) orientation.

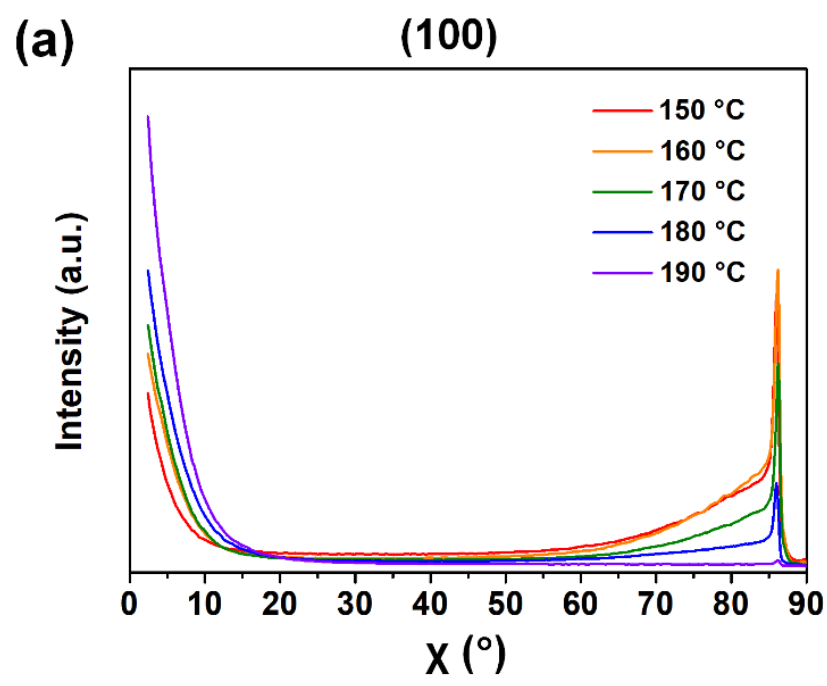

(b)

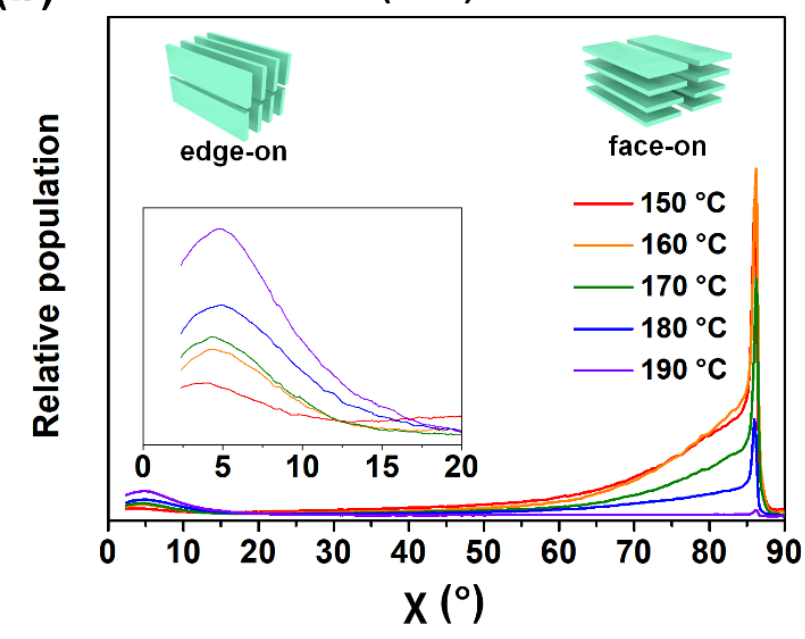

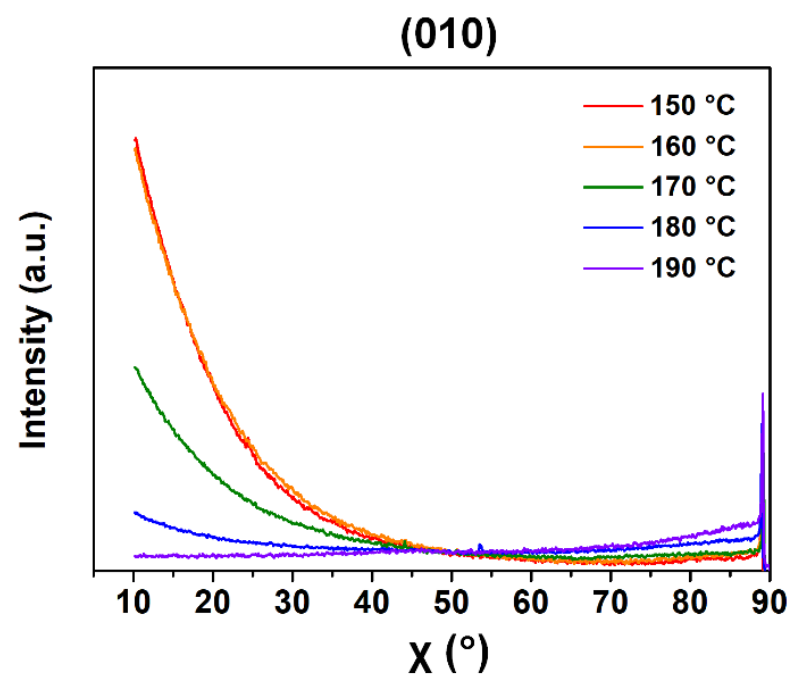

(010)

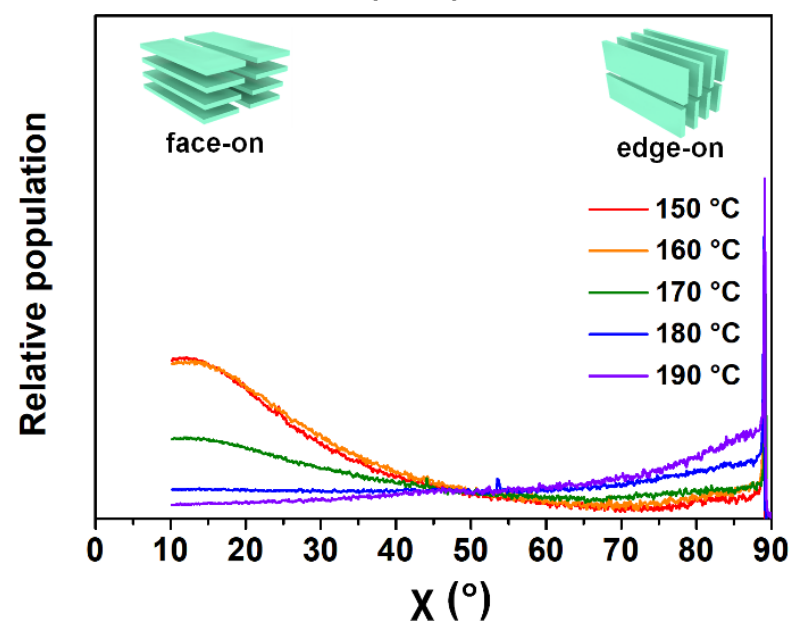


Figure S4. Illustrations of extracting the (100) and (010) peak intensities as a function of the pole angle $(\chi)$. Edge-on (or face-on) crystallites for the lamellar (100) peak are defined as crystallites with their lamellar stacking direction oriented within $30^{\circ}$ of the surface normal (or the in-plane direction), while for the $\pi-\pi$ stacking (010) peak with the $\pi-\pi$ stacking direction oriented within $30^{\circ}$ of the in-plane direction (or the surface normal).

\section{Edge-on}

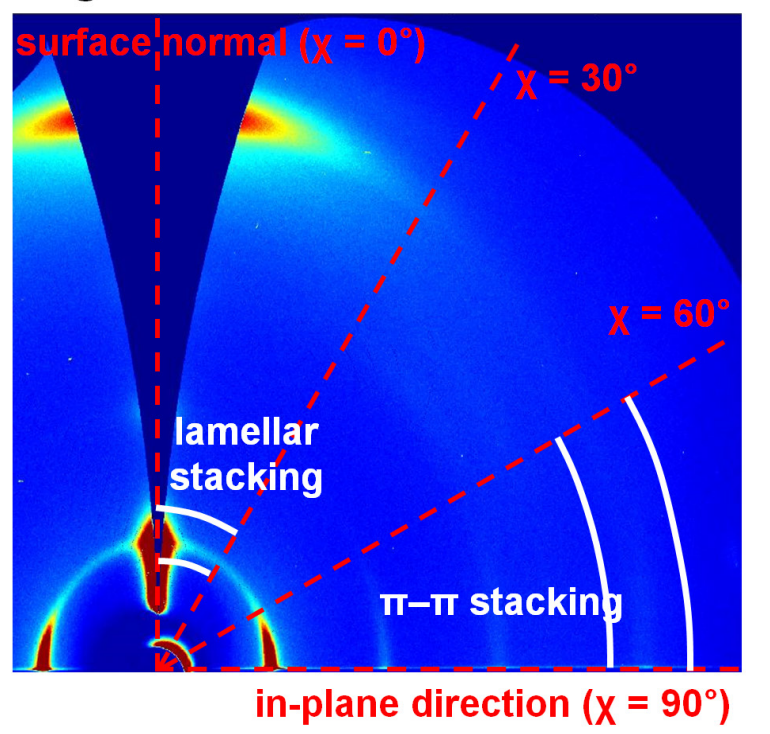

\section{Face-on}

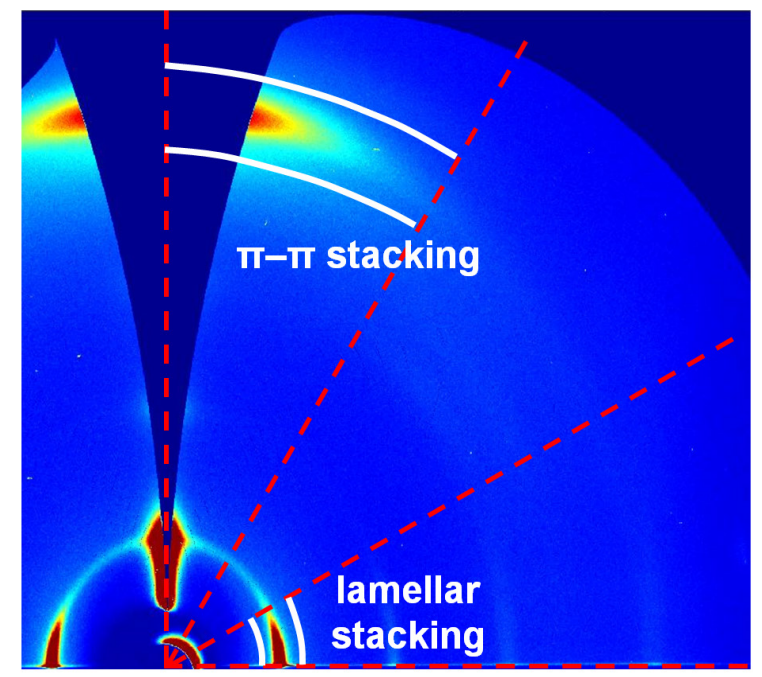


Figure S5. Out-of-plane and in-plane GIWAXS spectra of RP-3T extracted from 2D GIWAXS patterns of RP-3T as-cast film and annealed films at various temperatures.
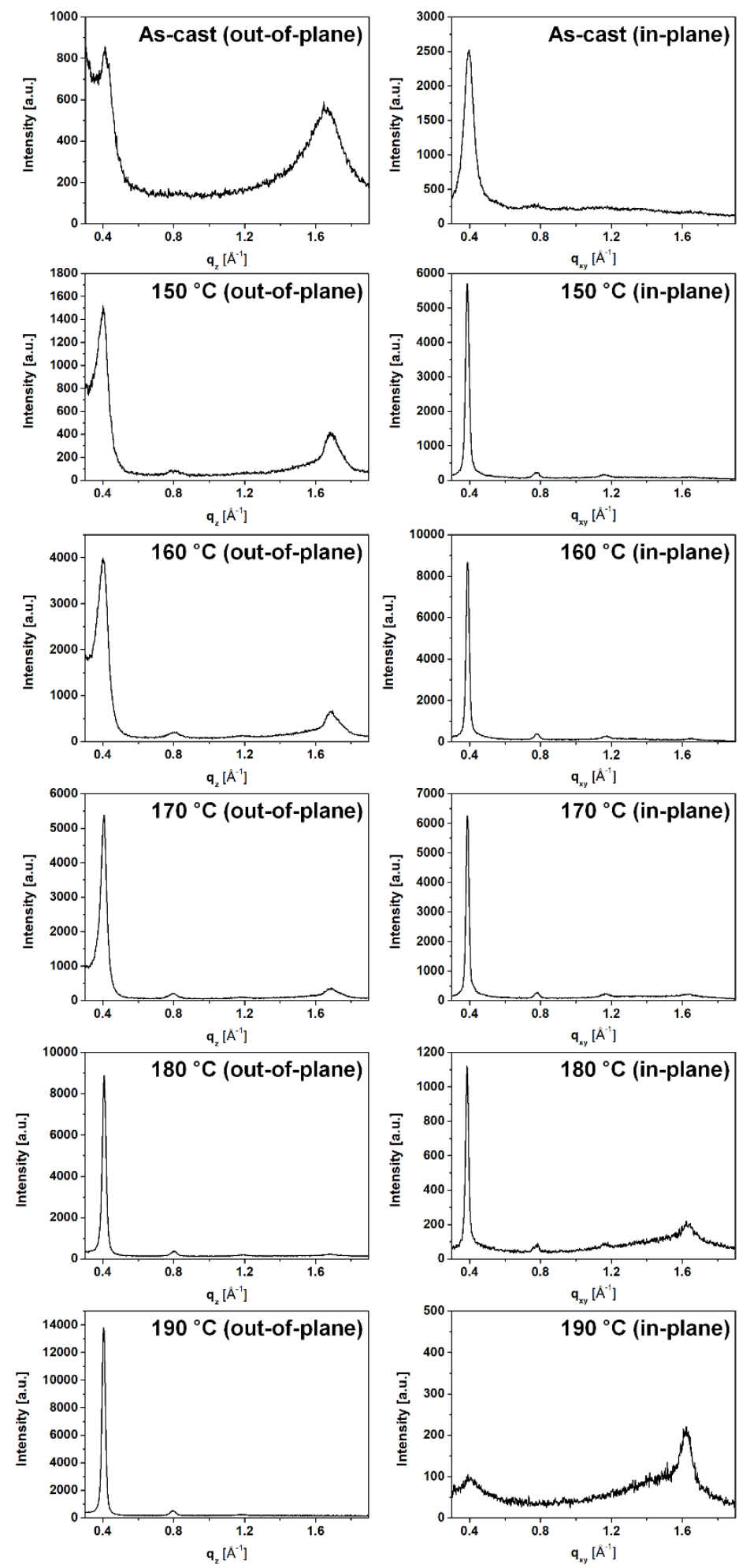
Figure S6. Angle-dependent NEXAFS spectra of as-cast and annealed P3HT and RP-3T films.
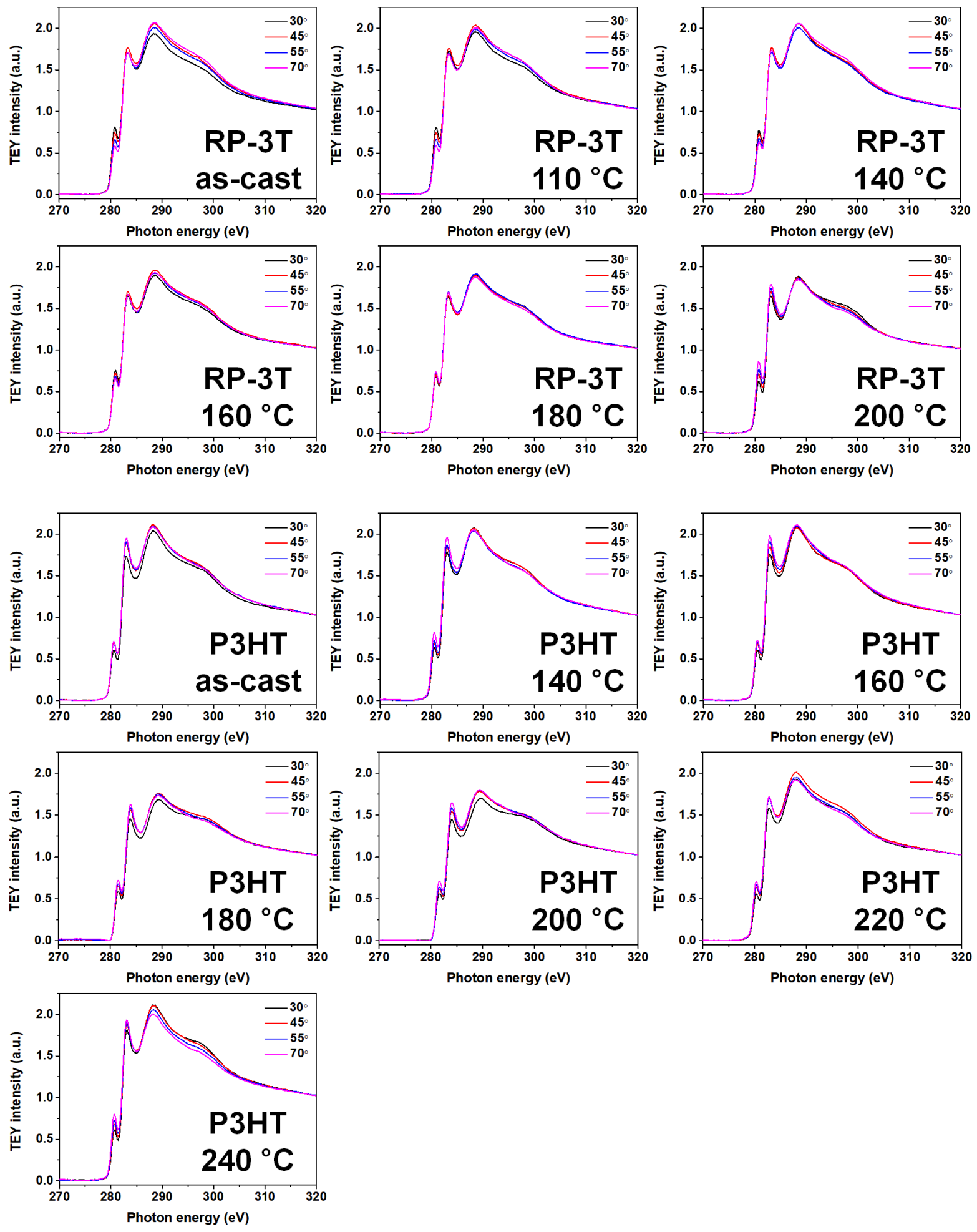
Figure S7. (a) Photos of water and glycerol droplets on RP-3T films. (b) Contact angles and (c) surface energies as a function of annealing temperature. Surface energies were determined according to the geometric mean equation, $(1+\cos \theta) \gamma_{\mathrm{pl}}=2\left(\gamma_{\mathrm{s}}^{\mathrm{d}} \times \gamma_{\mathrm{pl}}^{\mathrm{d}}\right)^{1 / 2}+2\left(\gamma_{\mathrm{s}}^{\mathrm{p}_{\mathrm{s}}} \times \gamma_{\mathrm{pl}}^{\mathrm{p}}\right)^{1 / 2}$, where $\gamma_{\mathrm{s}}$ and $\gamma_{\mathrm{pl}}$ are the surface energy of the sample and the probe liquid, respectively, and the superscripts $d$ and $p$ refer to the dispersion and polar components of the surface energy. ${ }^{1}$

(a)

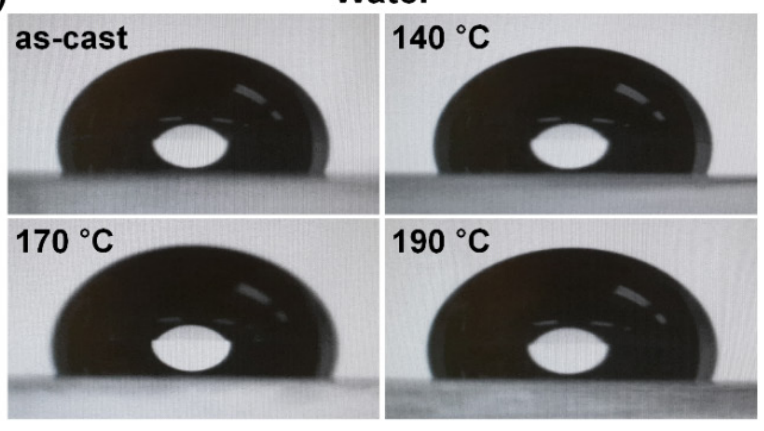

(b)

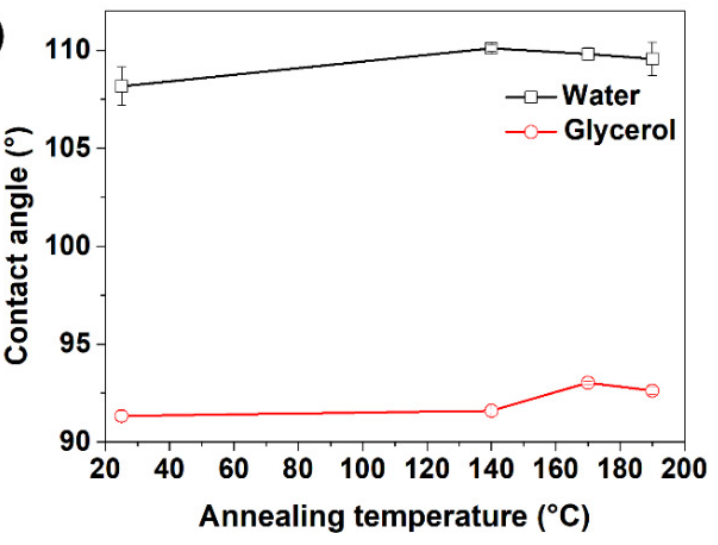

Glycerol

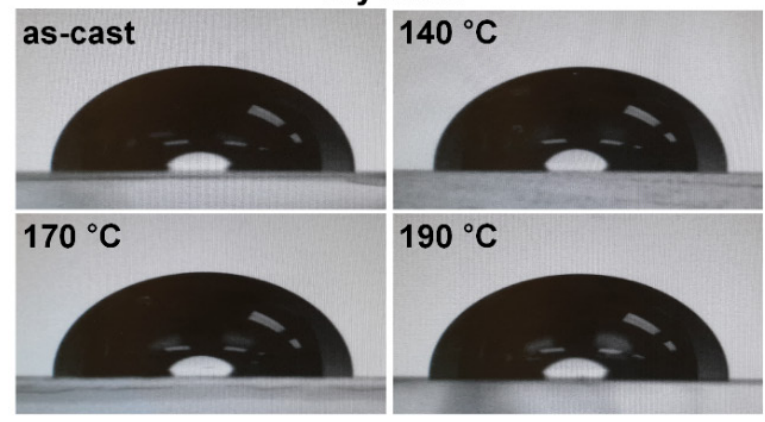

(c)

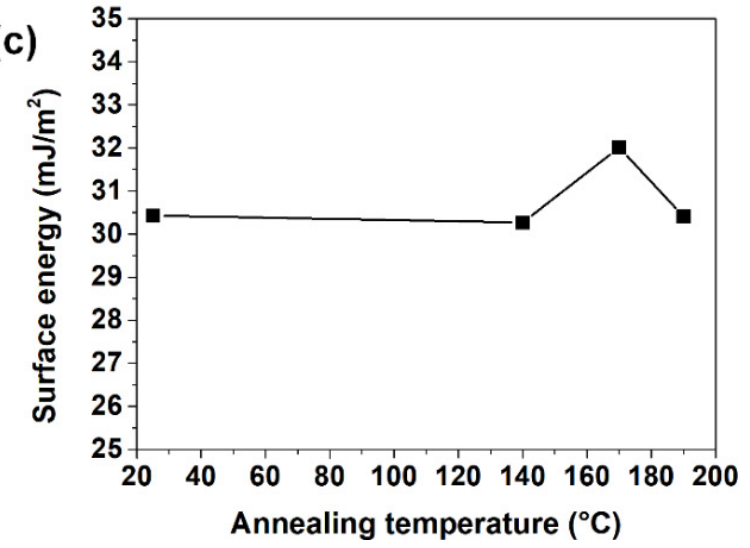


Figure S8. GIWAXS patterns of RP-3T annealed films coated on bare glass (top) or PEDOT:PSScoated glass (bottom). The films were annealed at 150, 160, 170, 180 and $190{ }^{\circ} \mathrm{C}$ for 10 min and cooled back to room temperature at ambient condition.
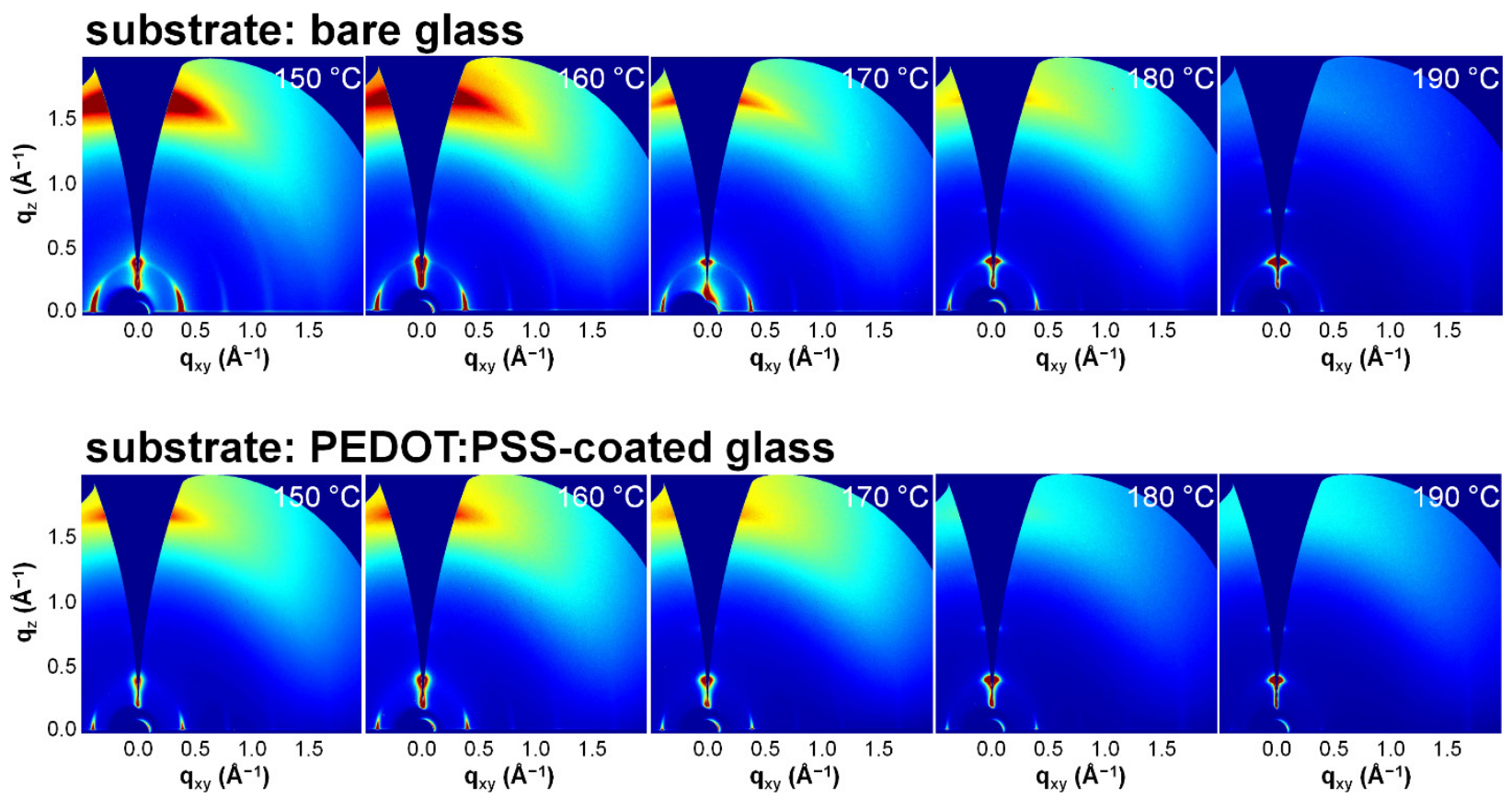

Table S1. Dichroic ratios of P3HT and RP-3T films measured via NEXAFS spectroscopy as a function of annealing temperature.

\begin{tabular}{ccc}
\hline $\begin{array}{c}\text { annealing } \\
\text { temperature } \\
\left({ }^{\circ} \mathrm{C}\right)\end{array}$ & \multicolumn{2}{c}{ Dichroic ratio, $R$} \\
\cline { 2 - 3 } no annealing & $0.14 \pm 0.01$ & $\mathrm{RP}-3 \mathrm{~T}$ \\
110 & $-^{a}$ & $-0.25 \pm 0.00$ \\
140 & $0.15 \pm 0.01$ & $-0.24 \pm 0.00$ \\
160 & $0.15 \pm 0.02$ & $-0.15 \pm 0.00$ \\
180 & $0.17 \pm 0.01$ & $-0.10 \pm 0.00$ \\
200 & $0.18 \pm 0.01$ & $0.07 \pm 0.00$ \\
220 & $0.21 \pm 0.01$ & $0.23 \pm 0.02$ \\
240 & $0.22 \pm 0.01$ & $-^{a}$ \\
& & ${ }^{a}$ \\
\hline
\end{tabular}

${ }^{a}$ Not measured. 
Table S2. $d$-spacings of lamellar and $\pi-\pi$ stacking in out-of-plane and in-plane directions in P3HT films as a function of annealing temperature.

\begin{tabular}{|c|c|c|c|c|}
\hline \multirow{3}{*}{$\begin{array}{l}\text { annealing } \\
\text { temperature } \\
\left({ }^{\circ} \mathrm{C}\right)\end{array}$} & \multicolumn{4}{|c|}{$d$-spacing $(\AA)$} \\
\hline & \multicolumn{2}{|c|}{ out-of-plane direction } & \multicolumn{2}{|c|}{ in-plane direction } \\
\hline & Lamellar packing $^{a}$ & $\pi-\pi$ stacking $^{b}$ & Lamellar packing $^{a}$ & $\pi-\pi$ stacking $^{b}$ \\
\hline no annealing & 16.22 & 3.77 & 17.00 & 3.88 \\
\hline 150 & 15.94 & 3.75 & 17.13 & 3.88 \\
\hline 200 & 15.71 & 3.74 & 16.89 & 3.88 \\
\hline 220 & 16.05 & - & 17.01 & 3.87 \\
\hline
\end{tabular}

${ }^{a}$ Corresponding to the (100) peak in the GIWAXS pattern. ${ }^{b}$ Corresponding to the (010) peak in the GIWAXS pattern.

\section{References}

1. Kang, B.; Lim, S.; Lee, W.H.; Jo, S.B.; Cho, K. Work-Function-Tuned Reduced Graphene Oxide via Direct Surface Functionalization as Source/Drain Electrodes in Bottom-Contact Organic Transistors. Adv. Mater. 2013, 25, 5856. 